\title{
Learning Math for 1st Grade Primary School Students using Intelligent Tutoring Systems
}

\author{
Nadiran Amanda ${ }^{a}$, Felix Andersen ${ }^{a}$, Ryan Christian ${ }^{a}$, Harco Leslie Hendric Spits Warnars ${ }^{b^{*}}$, Arief \\ Ramadhan $^{b}$, Arman Syah Putra ${ }^{b}$, Nurulhuda Noordin ${ }^{c}$ and Wiranto Herry Utomo ${ }^{d}$ \\ ${ }^{a}$ Computer Science Department, School of Computer Science, Bina Nusantara University, Jakarta, Indonesia \\ 11480 \\ ${ }^{\mathrm{b}}$ Computer Science Department, BINUS Graduate Program - Doctor of Computer Science, Bina Nusantara \\ University, Jakarta, Indonesia 11480. \\ ${ }^{c}$ Centre for Information Technology Studies, Faculty of Computer and Mathematical Sciences, Universiti \\ Teknologi MARA (UiTM), Shah Alam, Malaysia 40450. \\ ${ }^{\mathrm{d}}$ Department of magister science of information technology, Faculty of Computing, President University, Jawa \\ Barat, Indonesia 17550.
}

Corresponding author: Harco Leslie Hendric Spits Warnars email: spits.hendric@ binus.ac.id

\section{Article History: Received: 10 November 2020; Revised 12 January 2021 Accepted: 27 January} 2021; Published online: 5 April 2021

\begin{abstract}
Primary education after early childhood education is an important momentum in the education stage of a human being, in this case, education at the grade 1 level of elementary school. Where the delivery of subject matter properly and correctly at the early stages of this primary education will affect human education in the future. As well as the importance of early education at the elementary level, mathematics is an important initial subject to be given properly and correctly, so that the specter that says mathematics is a difficult subject is not true when a student in the early stages of basic education gets a good and correct mathematics education. Moreover, The Covid19 pandemic period is one of the parameters of the difficulty of knowledge transfer for students in the early stages of basic education, where physical meetings are not allowed to avoid Covid19 transmission in the classroom. Therefore, integrated tutoring systems (ITS) are a knowledge transfer tool that can help teachers, students, and parents in the delivery of subject matter, especially mathematics subjects which have a track record of being a scourge, scary and difficult to learn. In this paper, a simple ITS specifically for tutoring mathematics lessons for grade 1 elementary school students in Indonesia is tried to be implemented using the Unified Modeling Languages (UML) tool as an object-oriented modeling tool. Use case diagrams are applied to describe business processes in the ITS system that we built and use class diagrams modeled database table designs. Not to forget, the user interface (UI) from the results of the implementation prototype was displayed as a communication medium for users, both children, and tutors, in using applications built using Personal Home Pages (PHP) and MySQL.
\end{abstract}

Keywords: Intelligent tutoring systems, math intelligent tutoring systems, interactive learning, math for elementary school, math tutoring systems, mathematics intelligent tutoring systems.

\section{Introduction}

Humans as part of an almighty creation that was created in the image of their creator, are a creation that was created from the very beginning to rule the earth and everything in it. As a noble creation who is empowered to rule the earth and everything in it more wisely, it is important that humans master knowledge by carrying out the teaching and learning process (Pai et al., 2020). The covid19 pandemic incident is evidence of human greed by creating biological weapons that use pathogens, such as viruses, bacteria, or disease-producing organisms to control other humans. In the end, it is because of human greed and negligence that it becomes a weapon for the masters to eat, destroying the earth's ecosystem in a prolonged manner, hitting all areas of human life such as education, economy, arts and culture, and others. Blaming animals as the cause of the COVID-19 pandemic is evidence of human greed where animals, as almighty creatures, are also victims. It took years for this earth to return to normal following the adaptation of the earth and everything in itself as an omnipotent creation (Alkhatlan \& Kalita, 2018).

Humans as noble creatures are created to learn and always learn so that the earth and everything in it can be managed properly and wisely, even though many smart people are untrue and unwise. Therefore, teaching and learning activities are always a crucial moment in human life, which starts from the earliest stages, 
namely at an early age or in kindergarten (Sette et al., 2016). However, at this stage of early childhood education, most of this early childhood is more directed to play and socialize more so that they train their motor activities and build communication with the surrounding environment. Basic education after early childhood education is an important momentum in the education stage of a human being, so that when a person experiences a good and appropriate early stage of basic education, the beginning of this basic education will be good and pleasant for a human being to experience knowledge transfer experience as part of education (Rus et al., 2015). On the other hand, if humans experience trauma and unpleasant events in the early stages of basic education, then after that they can be sure that they will not get used to it and will not enjoy the joys of getting an education. Of the many materials that must be given to students in the early stages of basic education, in this case, grade 1 elementary school students, is a mathematics subject (Chughtai et al., 2015).

In order to prepare great national leaders and prepare human resources who can compete with other nations to become a developed Indonesian state, post Gen $\mathrm{Z}$ generation education must be prepared as early as possible. Moreover, it is equipped with the use of technology as early as possible in learning something so that they are familiar with the use of this technology. However, in Indonesia, education for post Gen $\mathrm{Z}$ creation, which amounts to $10.88 \%$ of Indonesia's total population of 270.20 million people, has not been fully carried out by the government and mostly carried out by the private sector, which in this case differs in the way of teaching and delivery (Jiménez et al., 2018).

The current condition of the world makes it almost impossible for many activities to be held normally, it is because of the ongoing pandemic, caused by the Corona Virus in late 2019, up until now. Since the Virus has continued to claim lives in many countries, it is risky and dangerous especially for the elder and children with weak immunity. One of many activities hampered by this pandemic is of course teaching and learning activities, where many places held them by online learning technique using video conference platforms such as Zoom or Google Classroom (Kulik \& Fletcher, 2016).

In an early learning environment, it is especially hard to provide a good learning experience, especially without face-to-face contact with the students themselves, where a teacher can give an immense amount of attention and observation to the students' behavior (Malekzadeh et al., 2015). With this ability to tend to the student directly almost entirely removed because of the online learning barrier, it will affect the process of learning on each student. Because of this inconvenience, our group decided to write a research paper about intelligent tutoring systems, specifically for Math subject, hence the research title "Learning Math for 1st Grade Primary School Students using Intelligent Tutoring Systems”.

Math Intelligent Tutoring Sytems that we make will focus more on the $1^{\text {st }}$ Grade Primary School, because they need teachers who can teach them basic mathematics learning, different from the upper class who can begin to use their logic to start thinking about how to solve mathematics because they have learned the basics of it before. Alongside video conference platforms for online learning by the teacher, students in $1^{\text {st }}$ Grade can use intelligent tutoring system tools for independent learning or as supplementary to the school lesson (Mousavinasab et al., 2018). We can learn and compare the effectiveness of an intelligent tutoring system with a traditional (peer-to-peer) tutoring system, and visualize the end product by creating the use case diagram, class diagram, and the user interface of the product, which is a web-based intelligent tutoring system.

\section{Previous Research}

The proposal for the idea of an intelligent guidance system that we designed is made web-based that takes into account efficiency and effectiveness, which is based on previous research by Kulik et al., That with controlled evaluation, the intelligent tutoring system is proven to be able to improve student performance when stacked up against traditional classrooms. (Brawner \& Gonzalez, 2016). Another factor is effectiveness and efficiency and has also been described in research conducted by Almasri, A et al., That the survey conducted from 2000 to 2018 produced major progress in the knowledge transfer process as part of the teaching and learning process. In addition, the application of using various media can ultimately contribute to education from all parties, be it, teachers, students, and parents, where the ITS implementation process motivates students to learn independently and sustainably. Besides, previous research in 2017 was carried out by Nour N AbuEloun and Samy S Abu Naser, where they created an intelligent math tutoring system for selected students, showing efficient and positive results and great building the teaching and learning community (Nye et al., 2018). Moreover, there are several works based on previous research, the context of ITS, such as from another research, Bartelet et al studied the effectiveness of an ITS, and shows that the tools improve the general skills of the student, but with the outcome needing more touch on the guidance of the teacher to also motivate the student to learn (Bartelet et al., 2016). 
Meanwhile, research in the field of Intelligent Tutoring Systems (ITS) is a research that has been done a lot where there were 1,740 published papers throughout the year if we do a search using google scholar with a search for the term "intelligent tutoring system" (allintitle: "intelligent tutoring system") or (allintitle: intelligent tutoring system) in the title of the paper (Abueloun \& Naser, 2017). However, ITS in the field of math or mathematics is still very rare where if you use google scholar a search is made on published papers throughout the year which only search for math words and "intelligent tutoring system" in the title of the paper (allintitle: Math "intelligent tutoring system") or (allintitle: Math intelligent tutoring system) there were only 6 published papers with the oldest paper in 1996 and for the search for the words "intelligent tutoring system" and mathematics in the title of the paper (allintitle: mathematics "intelligent tutoring system") or (allintitle: mathematics intelligent tutoring system) there were only 41 published papers with the oldest paper in 1989 (Almasri \& Ahmed, 2019). The next search was a search for the Indonesian language published papers using the words "matematika" as a translation of mathematics in Indonesian and "intelligent tutoring system" in the title of the paper on google scholar (allintitle: matematika intelligent tutoring system) then the result was only 1 published paper in 2020 (Akkila et al., 2019).

In accordance with the description in the previous paragraph, the use of ITS for learning mathematics is still very rare and this is an opportunity for this research to be carried out. The following are some of the research that has been and is being carried out in the application of ITS for learning mathematics. Starting from research conducted by Zhang et al. where ITS is used to help elementary and middle school students in learning show amazing results where students who are equipped with ITS in learning mathematics have satisfactory test scores compared to students who do not use ITS (Zhang \& Jia, 2017).

In the research proposed by Mondragon et al, for learning mathematics, an experiment was carried out comparing students who were divided into 2 groups with six students each using ITS and not using ITS and the results showed that the selection of tutors was very important in supporting student success. in using ITS. The assistance of a tutor, which in this case the teacher can represent is an added value where the role of the teacher as a parent in the school is one of success, where sometimes students do not get the support and attention of their biological parents because the parents are preoccupied with their duties. their job as parents is to take care of the house and earn income and are ignorant of the world of education (Mondragon et al., 2016).

Other previous research involving the use of a math-smart tutoring system was also carried out by Bringula et al, by conducting experiments that were divided into two groups, where the experimental group with access to an intelligent guidance application system called MathCal, and a group that did not use ITS (Bringula et al., 2016). The results of this experiment concluded that the ITS used in the experiment to improve overall student performance in problem-solving shows a positive and encouraging result which supports and encourages students to be able to learn mathematics independently. Also, Walkington et al tried to personalize mathematical problems by matching students' interests using the help of an intelligent guidance system application. This experiment also utilizes selected students to solve several maths problems, either normal problems or personal problems which are of course given as part of the level of education that the student is taking. The results show that higher overall performance is seen in personalized problems, which in turn can confirm the advantages of using an intelligent guidance system which essentially has a major influence on students in understanding and studying mathematics (Walkington \& Bernacki, 2019).

Furthermore, research conducted by Huang et al. shows that the use of the ITS application called ALEKS (www.aleks.com) for learning mathematics for sixth-grade elementary school students in the USA is a big stepping stone for the student that can be done after school hours and is an additional subject matter. This study raises the use of ITS to improve understanding and learning mathematics by paying attention to three parameters, namely race or ethnicity, gender, and socioeconomic status and the results show that there is equality of results in terms of classes taught by teachers of different ethnicities and in this case gender and status does not affect (Huang et al., 2016). Besides, the online guidance system that utilizes ITS to help elementary school students in learning subjects has always been a scourge for students in every consultation opportunity held by counseling teachers to elementary school students. This study uses data from 572 elementary school students and the data is processed using a statistical approach, namely the Principal Component Analysis (PCA) method using thirteen variables compared to the results of a survey built on a 
scale of 1 to 5 to identify four components of motivation. namely mood, outcome, attitude. and declarative. This study found that there is a good relationship between motivation and online guidance for mathematics subject matter which is divided into three levels of approach in implementing ITS (Tywoniw et al., 2020).

\section{Proposed Model}

In our proposed model, we design to use a use case diagram shown in figure 1 as a representation of the business processes displayed on the model and use a class diagram shown in figure 2 represented as a relationship between classes where each class is represented as a database table. In addition, the User Interface (UI) shown between Figures 3 to 10 are displayed as a prototype display for communication between the user and the application.

As seen in the use case diagram in Figure 1, there are three actors, namely users, tutors, and admins including eight use case activities. Starting with the use case activity register where each user must register before they can use the system, where they must enter user data such as user name, Name, phone number, date of birth, gender, address, email, and password where these attributes can be seen in the user class as seen in Figure 2. Figure 4 shows the User interface (UI) for the registration process. The login use case will be used for users who have registered and can enter the system by entering their email address and password where the UI can be seen in figure 5. The system will check the stored database to check if the entered data matches the saved login data. If they match, the user can log in to the system and if they don't match, the system will give a warning indicating that the user does not have access to the system and also provides a link if they forget their email address and password. In this case, only dealing with cases if they forget the password while cases of forgetting the email address cannot be handled, wherein the case of forgetting the email address they have to load a new account. In the link to forget login data such as email address and password, the user must enter the registered email address and the system will send a link to update their password. Then the user opens an email containing the contents to update the password and updates the password which will be updated to the system.

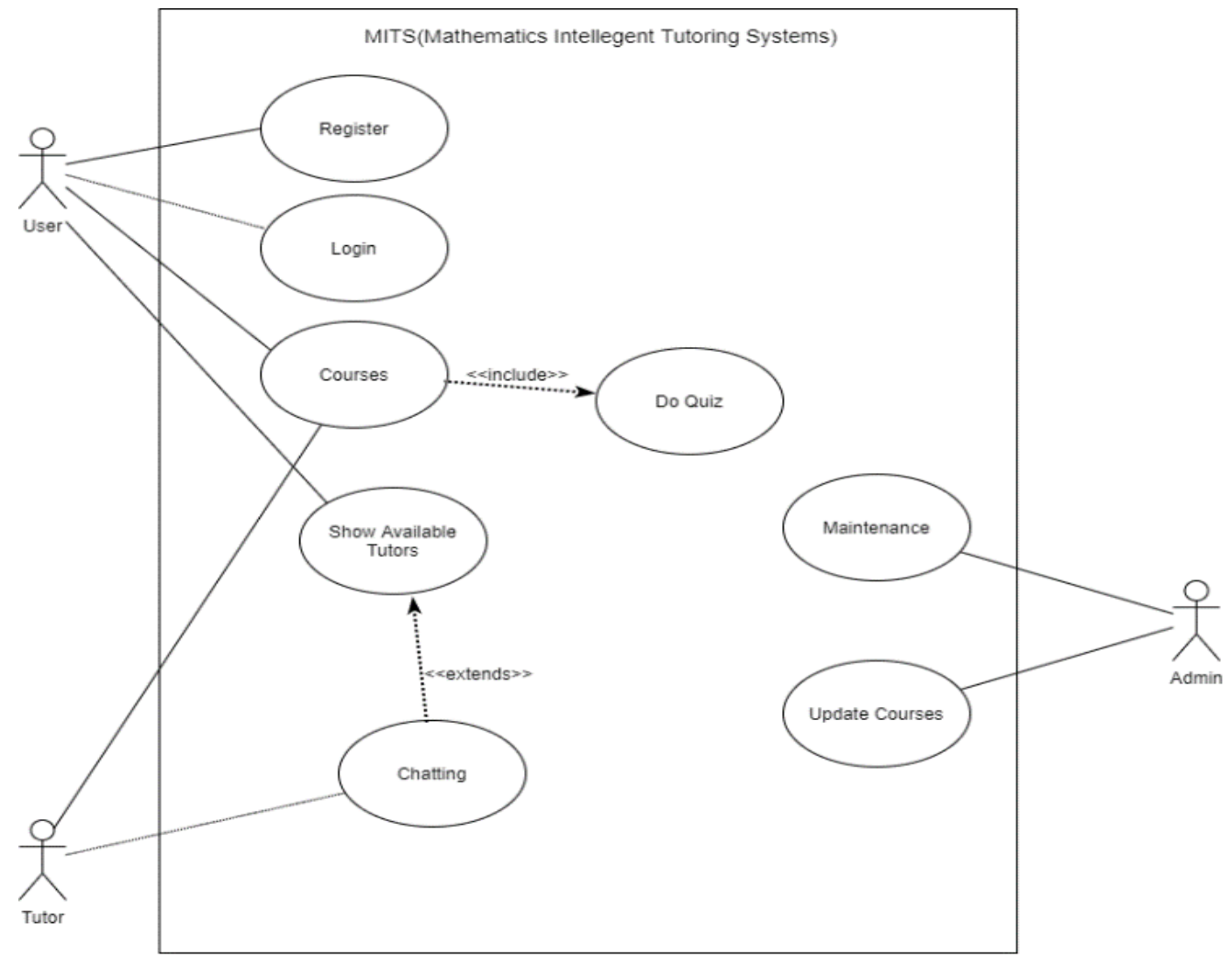

Figure 1. MITS Use case diagram

In the next use case activity, namely use case "courses", the user can choose which math topic he wants to study as seen in UI Figure 6. For the record, the UI in this paper is in Bahasa because this application is implemented for elementary school students in Indonesia. Use case "do quiz" is a sub use case of use case "course" which has a relation <<include〉> which means "do quiz" must be done when working on use case 
"course". Figure 7 shows the UI for an example of answering a quiz on one of the selected math subject topics and where previously the user will have Figure 8 as a UI with a choice of three types of problemsolving options written in Bahasa "mudah", "sedang", and "sulit" which means in English is "easy", "medium", and "difficult". Moreover, figure 9 shows an example of doing a quiz selection categorized as easy or "mudah" in Bahasa.

Meanwhile, chat use cases are represented as communication between users and there are the possibility of selecting a use case "show available tutors" where users can communicate with tutors who can be represented by their teachers in the class. Communication between users and tutors can be represented by parents of students who, in this case, want to get advice on math lessons for their children who are still in grade 1 elementary school, where the ages of the children are between 6 and 7 years old. Figure 10 shows a chat page that shows the conversation between parents and tutors that can be represented by elementary school teachers for grade 1 students. Table chatting and ChatComm as seen in figure 2 as saving tables database for this chatting purposes. Furthermore, the admin actor can carry out maintenance in matters related to the continuity of this application and also has the task of updating the course by updating or entering course content including quizzes for each course content.

Figure 2 shows a class diagram as a representation of the relationship between classes or class relations where in this case there are 7 classes such as chatting, chatcomm, user, usercourse, courseQuiz, Quiz and CourseLevel where each class is represented as a database table which means there are 7 database tables and of course Figure 2 is shown as a database model diagram showing the relationships between tables database. This class diagram is formed based on the processes represented by use case activities in the use case diagram shown in Figure 1 whereas previously mentioned where the table or class user is a data storage place in the use case activity process such as registration and login, and in addition to the classes or tables chatting and ChatComm are used to store conversation communication data in chatting use case activities as shown in figure 1 .

Next, is the relationship between these classes in Figure 2 where the user class has a 1 to many relationship with the chatting class, which means that each user in the user class can do many chats where each chat comes from 1 user in the user class. Additionally, the user class has a 1 to many relationship to the ChatComm class which means each user in the user class can have multiple chat replies in the ChatComm class and each reply chat in the ChatComm class comes from 1 user in the user class. Furthermore, the chatting class has a 1 to many relationship with the ChatComm class which means that each chat in the chatting class can have multiple chat replies in the ChatComm class and each reply chats in the ChatComm Class for 1 chat in the chatting class.

Hereinafter, the CourseLevel class is a representation table of the mathematics curriculum in the form of a chapter or learning level for grade 1 elementary school students and the Quiz class is a representation of multiple-choice questions for each chapter or level of learning the mathematics curriculum for grade 1 SD contained in the class CourseLevel. Multiple choice questions are represented as attributes in the Quiz class in Figure 2 where the "question" attribute is a multiple-choice question, while the "optionA", "optionB", "optionC", and "optionD" attributes are alternative answers to multiple-choice questions and the "answer" attribute is the correct answer choice for attributes "optionA", "optionB", "optionC", and "optionD". CourseLevel class has a 1 to many relationship with the Quiz class as shown in figure 2, which means that each course level or chapter in the CourseLevel class has many multiple-choice questions in the Quiz class and each multiple-choice question in the Quiz class is for 1 course level or chapter in the CourseLevel class.

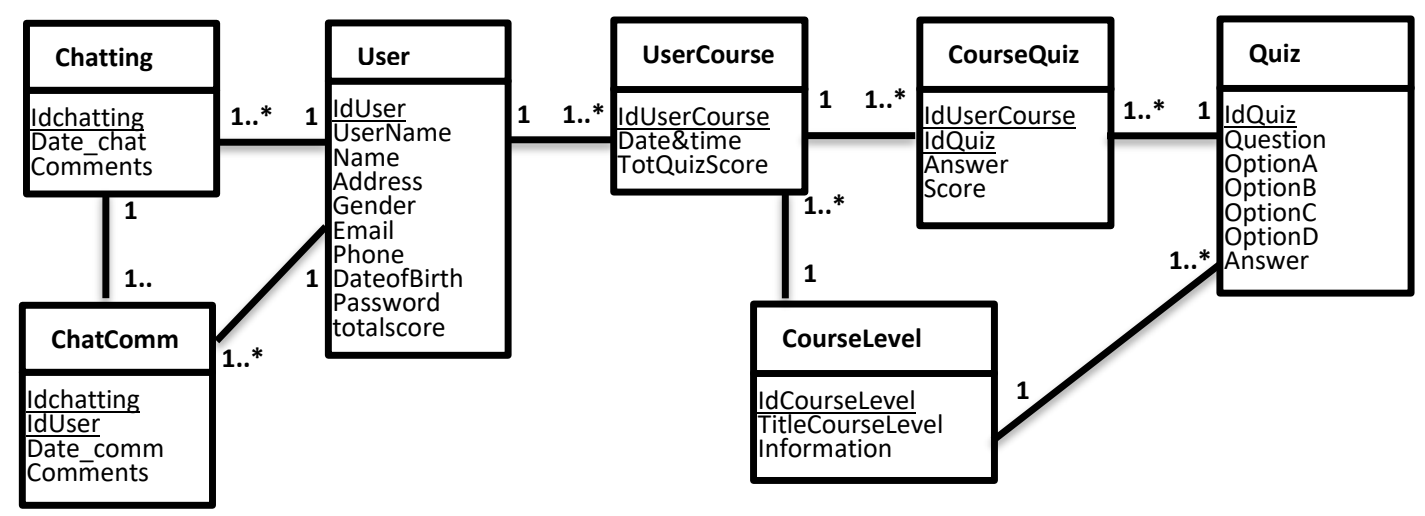

Figure 2. MITS Class diagram 
Meanwhile, the UserCourse and CourseQuiz classes as shown in Figure 2 are for transactions carried out by 1st-grade elementary school students in answering multiple-choice questions as a quiz, where this grade 1 elementary school student is shown as a user actor and this transaction is shown as a use case activity "do quiz" on the use case diagram in Figure 1. whilst the multiple-choice questions that will be done by students of primary school class 1 are in the Quiz class in Figure 2. The UserCourse class is used to record each time students work on a quiz question as shown as a "do quiz" use case activity in the use case diagram in Figure 1 by answering multiple-choice questions and the CourseQuiz class records the "answer" attribute of any answer for each multiple-choice question from The Quiz class and the "score" attribute will be filled with a score of 1 if the "answer" attribute in the CourseQuiz class matches the "answer" attribute in the Quiz class and will otherwise be filled with a score of 0 if the answer does not match.

The following is a description of the relationships between classes that are part of the "do quiz" use case activity which is shown in the use case diagram in Figure 1 which uses the UserCourse and the CourseQuiz classes. Starting from the UserCouse class which has a many to 1 relationship with the user and the CourseLevel classes, which means that each doing quiz in the UserCourse class is carried out by only 1 user as part of the user class and each doing quiz in the UserCourse class is part of 1 level or chapter of the course in the CourseLevel class. Hereafter, 1 user in the user class can work on many quizzes recorded in the UserCourse class, and 1 level or chapter of the course in the CourseLevel class can be carried out on many quizzes recorded in the UserCourse class. Moreover, the UserCourse class has a 1 to many relationship with the CourseQuiz class which means that every time doing a quiz in the UserCourse class has many answered quizzes as stored in the CourseQuiz class and each quiz answer in the CourseQuiz class is for 1 doing a quiz in the UserCourse class. Furthermore, the CourseQuiz class has many to 1 relationship with the Quiz class, which means that every quiz answer in the CourseQuiz class is to answer 1 question from the Quiz class, and 1 question from the Quiz class can be answered in many quiz answers in the CourseQuiz class.

Next is the User Interface (UI) as an implementation of the use case diagram in Figure 1, which uses a database as seen in the class diagram in Figure 2, and the UI in this paper is displayed in Bahasa because this application was developed using Personal Home Pages ( PHP) and MySQL databases are used by grade 1 elementary school students in Indonesia as previously mentioned. Figure 3 shows the UI main menu as the landing page of the application and on the landing page of MITS, the user will be greeted with several information, such as the headline of the MITS web application, the button to register now, navigation bar to view various information about this application.

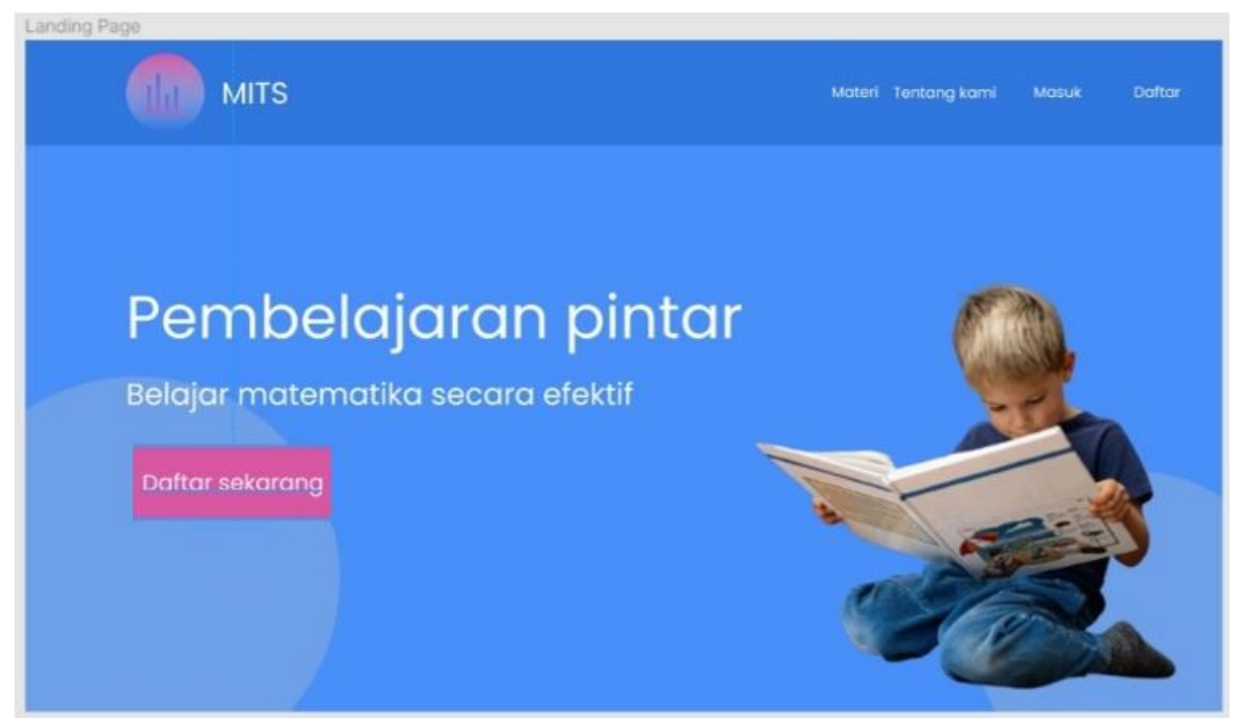

Figure 3. MITS User Interface for Landing Page 


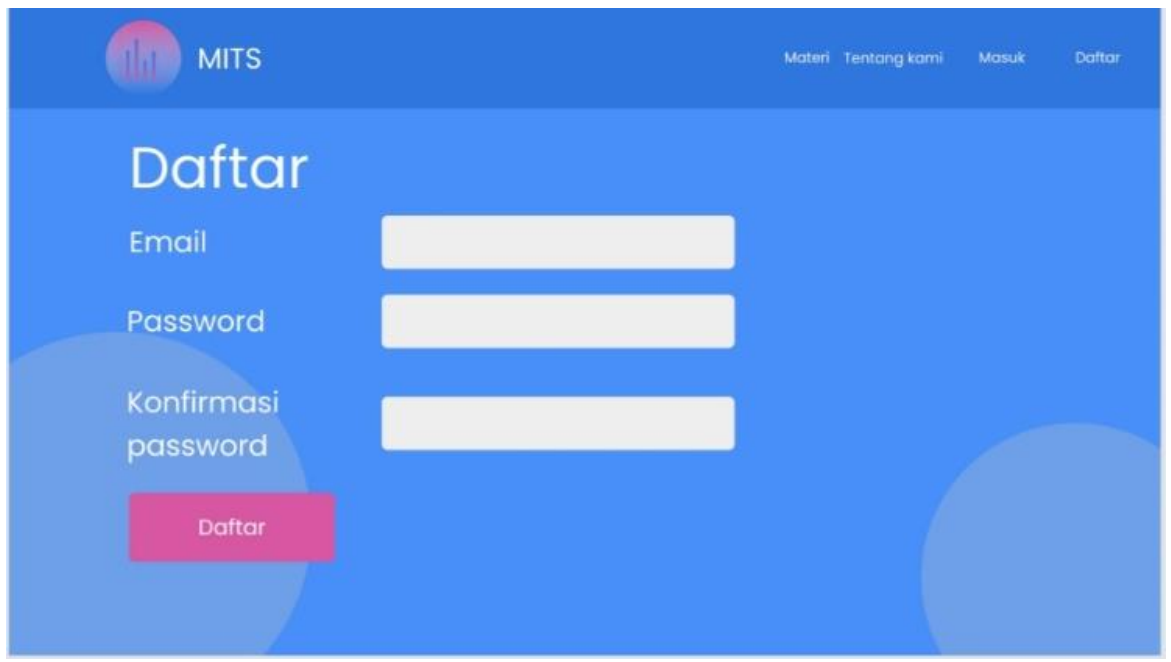

Figure 4. MITS User Interface for Registration Page

Figure 4 shows the UI menu for the registration process as seen in the use case diagram in Figure 1 where the data will be stored in the user table as shown in the class diagram in Figure 2. Moreover, figure 5 shows the UI menu for the login process as seen as well in the use case diagram in Figure 1 where the data will check the registered email address and password from the user table as shown in the class diagram in Figure 2. Every time a user forgets their email address and password, they will be refused entry to the system and must register a new one if they forget their email address and update their password every time they forget their password. In addition, if the input data for the email address and password match the data recorded in the user table as in the class diagram in Figure 2, the UI display will change as in Figure 6 where the user can select the math subject topic to be studied. Furthermore, on the course list page, as shown in Figure 6, the user can see the mathematics subject topic choices for grade 1 elementary school students and by clicking the "join" button, the user selects the topic option for that math subject and the UI menu page. will change to something as shown in the UI menu in figure 7 which is the UI menu for a particular topic of the selected math subject.

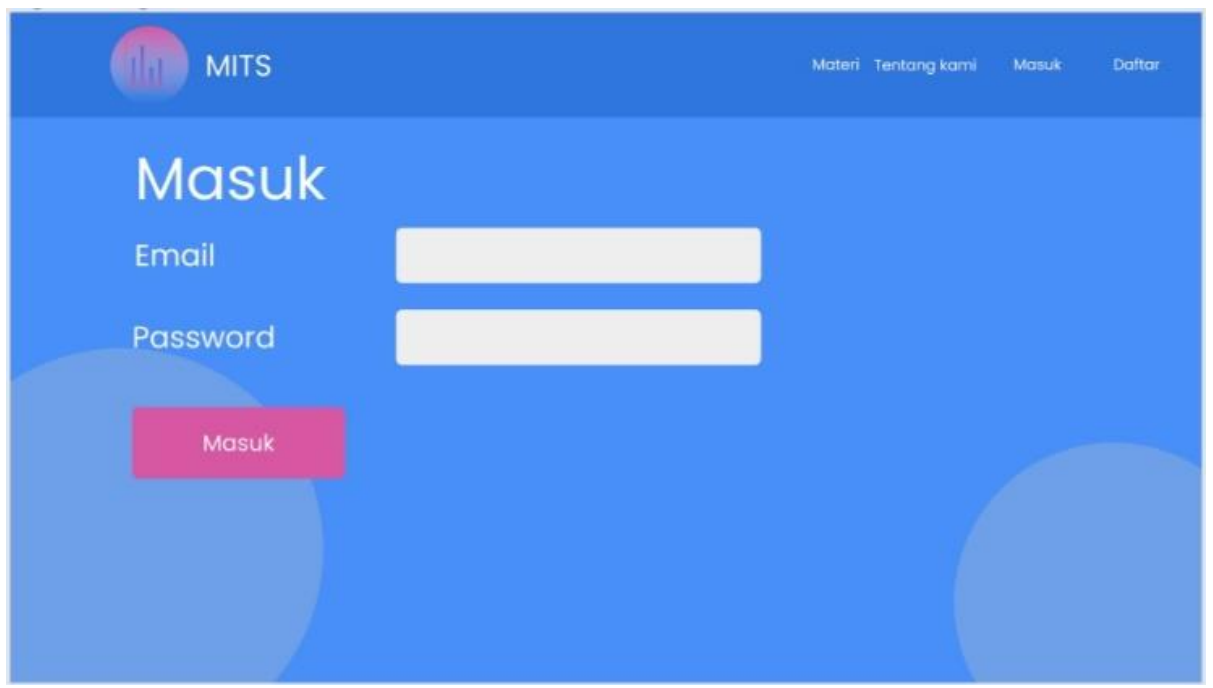

Figure 5. MITS User Interface for Login Page 


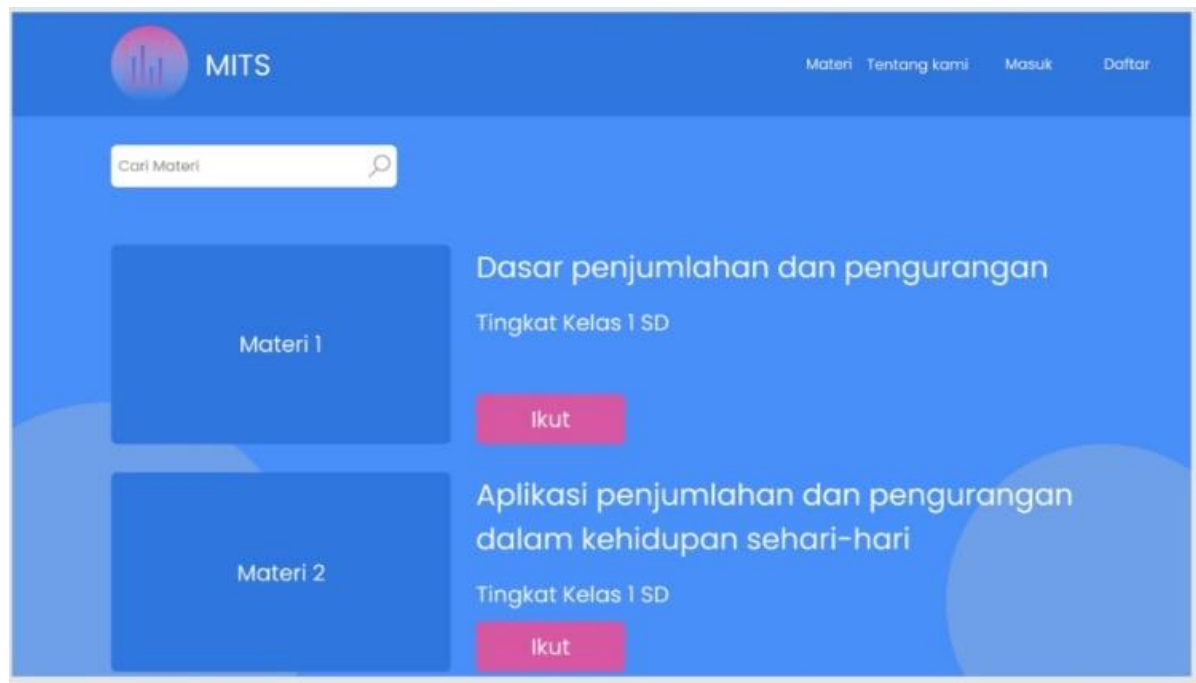

Figure 6. MITS User Interface for a Selected List Page of mathematics subject topics.

On the MITS single course page, as seen in figure 7, the user is greeted with video-based learning materials for each level of the lesson, with a clean user interface and also a playlist for selecting other video parts of video-based learning. Playlists and video-based learning content will be continuously updated according to the latest curriculum to ensure that every 1st grader of this elementary school gets the latest lessons and understanding of mathematics subjects. At the end of the shift in the learning page of the selected mathematics topic as shown in Figure 7, there will be an option for users or grade 1 elementary school students to choose to answer multiple-choice questions in order to test their mathematical skills at the level or math topic being studied. The UI menu for the multiple-choice questions has 3 categories as shown in Figure 8 with the choice words in Bahasa such as "mudah", "sedang", and "sulit" meaning easy, medium, and difficult in English.

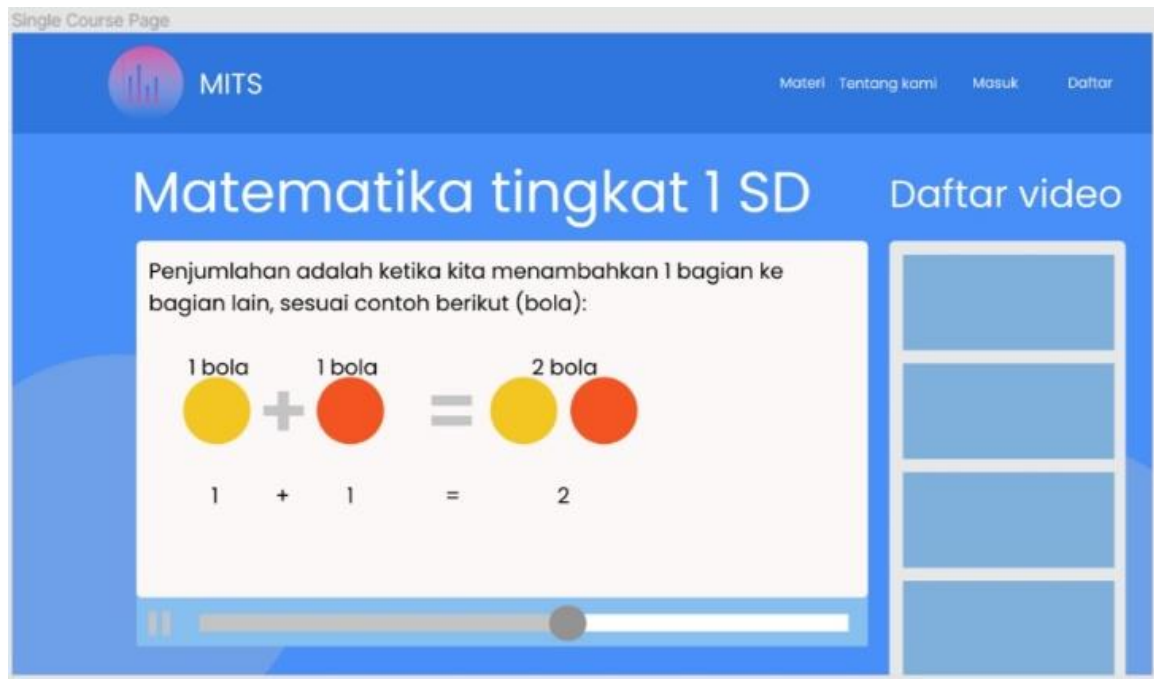

Figure 7. MITS User Interface for learning on one of the math subject topics. 


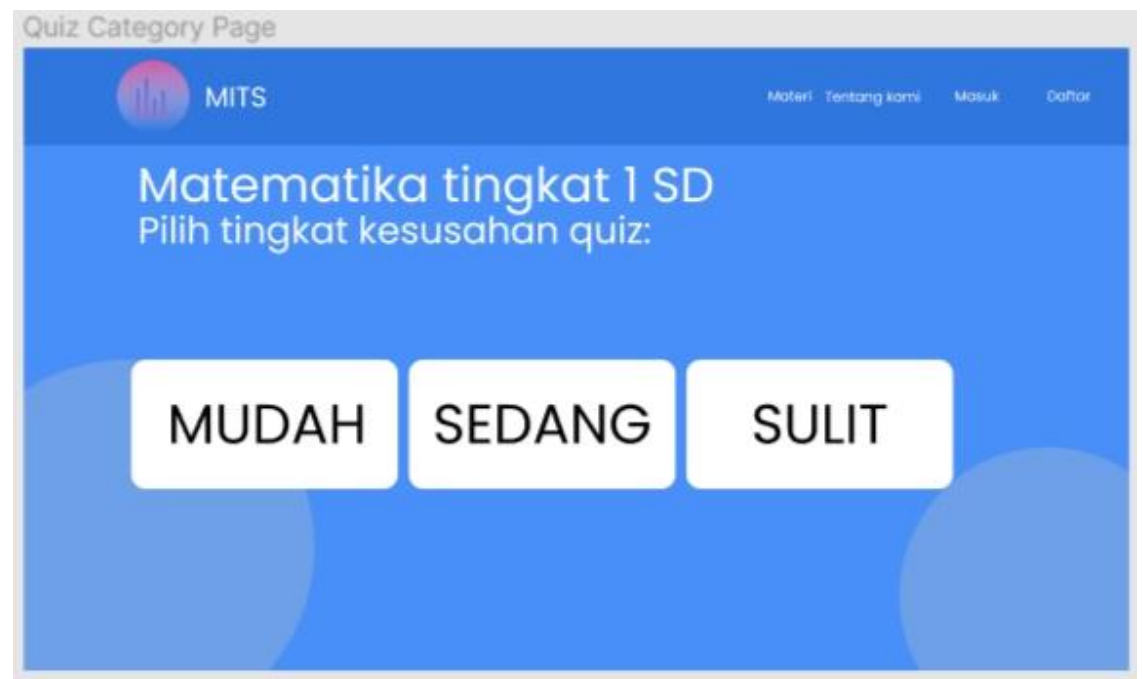

Figure 8. MITS User Interface for a choice of three different difficulty levels of the quiz to be done.

After the user selects a category to work on math topic questions that suit their abilities, the UI page display will change to a UI menu page for working on quiz questions as shown in Figure 9 wherein this case what is shown in Figure 9 is the work of multiple-choice questions that have a level "mudah" or easy in English. The process of working on questions as shown in Figure 9 will use a database table as shown in the class diagram in Figure 2, namely the UserCourse table to store data such as iduser, date and time of working on the quiz and the total score of the quiz score obtained and the level code of the level or the chapter studied refers to the CourseLevel table. Each quiz question that is displayed will be taken from the Quiz table which contains the following question and answers choices as multiple-choice questions and of course, the multiple-choice questions displayed are limited to questions whose designation corresponds to the level or chapter of the lesson being studied which refers to the CourseLevel table.

In addition, the CourseQuiz table will store each answer to each question answered by the user according to the question restrictions that are following the level or chapter of the lesson being studied. If the answer given by the user matches the answer in the Quiz table, the answer given by the user will have a score of 1 and the reverse will have a score of 0 if the answer is not suitable. The total of the assessment score from the activity of doing the questions for the specific level or chapter of the topic of this mathematics subject will be stored in the TotQuizScore attribute in the UserCourse table. Besides, the totalscore attribute in the user table will also be updated with the total score obtained.

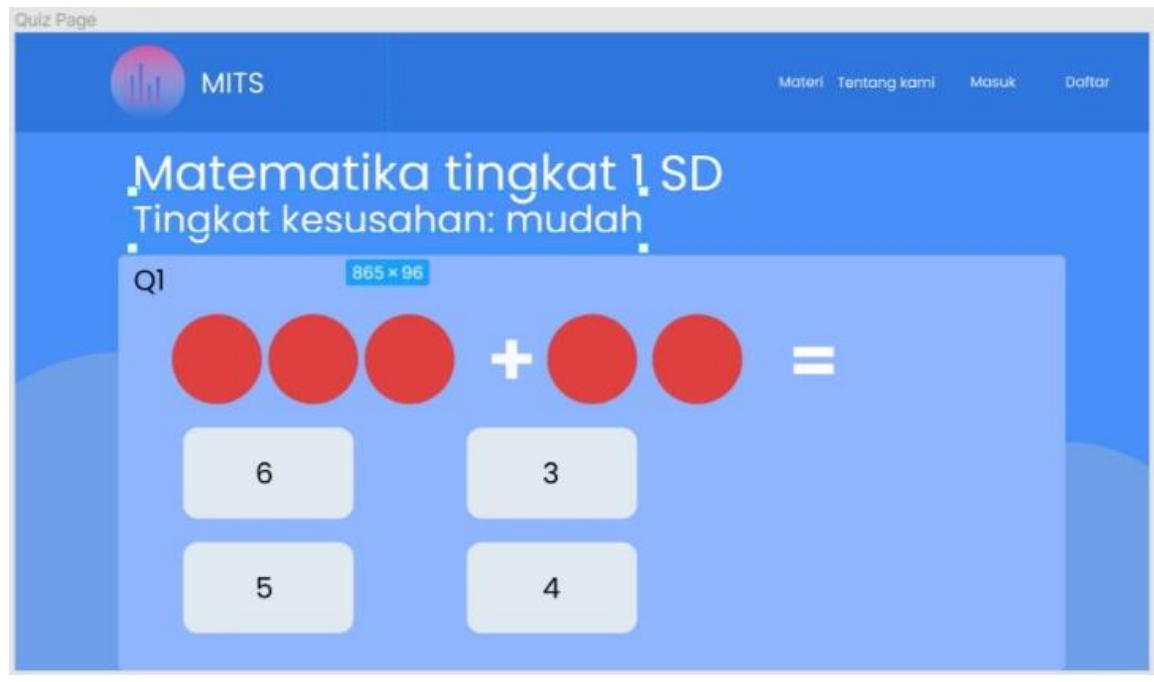

Figure 9. MITS User Interface for "mudah" or easy quiz examples of difficulty. 


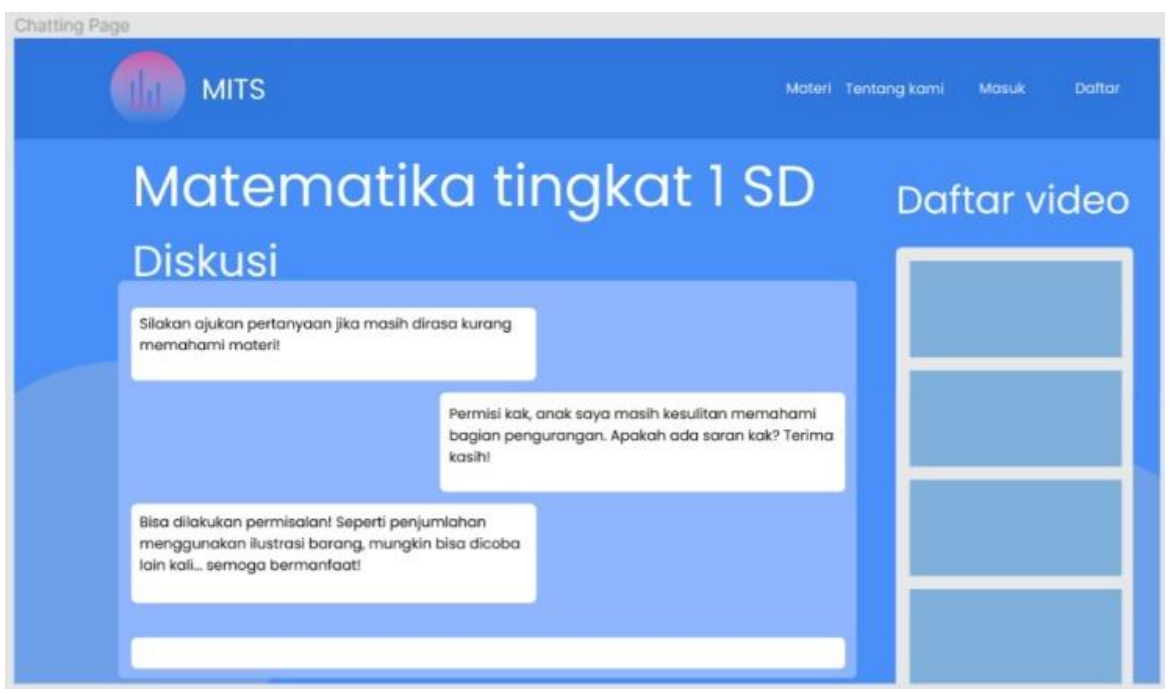

Figure 10. MITS User Interface for Chatting Page.

Lastly, figure 10 shows the UI menu for the chatting process as seen in the use case diagram in Figure 1 where the data will be stored in the Chatting and ChatComm tables as shown in the class diagram in Figure 2. This chatting process is used by parents when they are going to ask about the difficulties faced by their children who are still in grade $1 \mathrm{SD}$ when learning a topic of this math lesson. Student representation is represented by parents of students because grade 1 elementary school students are still aged between 6 or 7 years, so they need to be represented for discussions either with other parents or with the teacher who is the real tutor for math topics for 1 st-grade student of this elementary school.

\section{Conclusion}

The use of ITS for mathematics learning is expected to encourage interest in learning mathematics for grade 1 elementary school students, especially during the COVID19 pandemic where class meetings are not possible. Also, the use of ITS after the pandemic period has passed will strengthen and sharpen the ability of teachers in learning mathematics and especially for the 1st-grade students of elementary school and likewise parents who always pay attention to the education of their children. For future development, IT must be equipped with artificial intelligence (AI) technology such as the use of essay scoring which makes it easy to assess essay answers automatically. Besides, it is necessary that unstructured data on chat conversations need to be screened to find out what topics are often discussed by discussions between parents or between parents and teachers.

\section{Acknowledgments}

This work is supported by the Research and Technology Transfer Office, Bina Nusantara University as a part of Bina Nusantara University's International Research Grant contract number: No.017/VR.RTT/III/2021 contract date: 22 March 2021.

\section{References}

[1]. Abueloun, N. N., \& Naser, A. (2017). Mathematics intelligent tutoring system. International Journal of Advanced Scientific Research, 2(1), 11-16. www.allscientificjournal.com

[2]. Akkila, A. N., Almasri, A., Ahmed, A., Masri, N., Sultan, Y. A., Mahmoud, A. Y., Zaqout, I., \& Abu-naser, S. S. (2019). Survey of Intelligent Tutoring Systems Up To the End of 2017. International Journal of Academic Information Systems Research, 3(3), 71-81. www.ijeais.org/ijaisr

[3]. Alkhatlan, A., \& Kalita, J. K. (2018). Intelligent tutoring systems: A comprehensive historical survey with recent developments. ArXiv. https://doi.org/10.5120/ijca2019918451

[4]. Almasri, A., \& Ahmed, A. (2019). Intelligent Tutoring Systems Survey for the Period 2000- 2018. International Journal of Academic Engineering Research (IJAER), 3(5), 21-37. http://dstore.alazhar.edu.ps/xmlui/handle/123456789/456

[5]. Bartelet, D., Ghysels, J., Groot, W., Haelermans, C., \& Maassen van den Brink, H. (2016). The differential effect 
of basic mathematics skills homework via a web-based intelligent tutoring system across achievement subgroups and mathematics domains: A randomized field experiment. Journal of Educational Psychology, 108(1), 1.

[6]. Brawner, K. W., \& Gonzalez, A. J. (2016). Modelling a learner's affective state in real time to improve intelligent tutoring effectiveness. Theoretical Issues in Ergonomics Science, 17(2), 183-210.

[7]. Bringula, R. P., Basa, R. S., Dela Cruz, C., \& Rodrigo, M. M. T. (2016). Effects of prior knowledge in mathematics on learner-interface interactions in a learning-by-teaching intelligent tutoring system. Journal of Educational Computing Research, 54(4), 462-482.

[8]. Chughtai, R., Zhang, S., \& Craig, S. D. (2015). Usability evaluation of intelligent tutoring system: ITS from a usability perspective. Proceedings of the Human Factors and Ergonomics Society Annual Meeting, 59(1), 367371.

[9]. Huang, X., Craig, S. D., Xie, J., Graesser, A., \& Hu, X. (2016). Intelligent tutoring systems work as a math gap reducer in 6th grade after-school program. Learning and Individual Differences, 47, 258-265.

[10].Jiménez, S., Juarez-Ramirez, R., Castillo, V. H., \& Ramírez-Noriega, A. (2018). Integrating affective learning into intelligent tutoring systems. Universal Access in the Information Society, 17(4), 679-692.

[11].Kulik, J. A., \& Fletcher, J. D. (2016). Effectiveness of intelligent tutoring systems: a meta-analytic review. Review of Educational Research, 86(1), 42-78.

[12].Malekzadeh, M., Mustafa, M. B., \& Lahsasna, A. (2015). A review of emotion regulation in intelligent tutoring systems. Journal of Educational Technology \& Society, 18(4), 435-445.

[13].Mondragon, A. L., Nkambou, R., \& Poirier, P. (2016). Evaluating the effectiveness of an affective tutoring agent in specialized education. European Conference on Technology Enhanced Learning, 446-452.

[14].Mousavinasab, E., Zarifsanaiey, N., R. Niakan Kalhori, S., Rakhshan, M., Keikha, L., \& Ghazi Saeedi, M. (2018). Intelligent tutoring systems: a systematic review of characteristics, applications, and evaluation methods. Interactive Learning Environments, 1-22.

[15].Nye, B. D., Pavlik, P. I., Windsor, A., Olney, A. M., Hajeer, M., \& Hu, X. (2018). SKOPE-IT (Shareable Knowledge Objects as Portable Intelligent Tutors): overlaying natural language tutoring on an adaptive learning system for mathematics. International Journal of STEM Education, 5(1), 12.

[16].Pai, K.-C., Kuo, B.-C., Liao, C.-H., \& Liu, Y.-M. (2020). An application of Chinese dialogue-based intelligent tutoring system in remedial instruction for mathematics learning. Educational Psychology, 1-16.

[17].Rus, V., Niraula, N., \& Banjade, R. (2015). DeepTutor: An effective, online intelligent tutoring system that promotes deep learning. Proceedings of the AAAI Conference on Artificial Intelligence, 29(1).

[18].Sette, M., Tao, L., Gai, K., \& Jiang, N. (2016). A semantic approach to intelligent and personal tutoring system. 2016 IEEE 3rd International Conference on Cyber Security and Cloud Computing (CSCloud), 261-266.

[19].Tywoniw, R., Crossley, S. A., Ocumpaugh, J., Karumbaiah, S., \& Baker, R. (2020). Relationships Between Math Performance and Human Judgments of Motivational Constructs in an Online Math Tutoring System. International Conference on Artificial Intelligence in Education, 329-333.

[20]. Walkington, C., \& Bernacki, M. L. (2019). Personalizing algebra to students' individual interests in an intelligent tutoring system: Moderators of impact. International Journal of Artificial Intelligence in Education, 29(1), 58-88.

[21].Zhang, B., \& Jia, J. (2017). Evaluating an intelligent tutoring system for personalized math teaching. 2017 International Symposium on Educational Technology (ISET), 126-130. 